\title{
ON BILINEAR LITTLEWOOD-PALEY SQUARE FUNCTIONS
}

\author{
P. K. RATNAKUMAR AND SAURABH SHRIVASTAVA
}

(Communicated by Michael T. Lacey)

\begin{abstract}
In this paper, we study the bilinear Littlewood-Paley square function introduced by M. Lacey. We give an easy proof of its boundedness from $L^{p}\left(\mathbb{R}^{d}\right) \times L^{q}\left(\mathbb{R}^{d}\right)$ into $L^{r}\left(\mathbb{R}^{d}\right), d \geq 1$, for all possible values of exponents $p, q, r$, i.e. for $2 \leq p, q \leq \infty, 1 \leq r \leq \infty$ satisfying $\frac{1}{p}+\frac{1}{q}=\frac{1}{r}$. We also prove analogous results for bilinear square functions on the torus group $\mathbb{T}^{d}$.
\end{abstract}

\section{INTRODUCTION}

The theory of bilinear multipliers has been revived with the outstanding work of Lacey and Thiele [6], 7] on boundedness of the bilinear Hilbert transform.

Definition 1.1 (Bilinear Hilbert Transform). For $f, g \in \mathcal{S}(\mathbb{R})$, the bilinear Hilbert transform is defined by

$$
H(f, g)(x)=\text { p.v. } \int_{\mathbb{R}} f(x-y) g(x+y) \frac{d y}{y} .
$$

Writing the Fourier transforms for $f$ and $g$, the operator $H(f, g)$ takes the following form:

$$
H(f, g)(x)=-i \int_{\mathbb{R}} \int_{\mathbb{R}} \hat{f}(\xi) \hat{g}(\eta) \operatorname{sgn}(\xi-\eta) e^{2 \pi i x(\xi+\eta)} d \xi d \eta
$$

where

$$
\operatorname{sgn}(\xi)= \begin{cases}1, & \xi>0, \\ 0, & \xi=0, \\ -1, & \xi<0 .\end{cases}
$$

In [6], 7] Lacey and Thiele proved that

Theorem 1.2 (Lacey-Thiele Theorem [6, 7]). Let $1<p, q \leq \infty$ and $\frac{2}{3}<r<\infty$ be such that $\frac{1}{p}+\frac{1}{q}=\frac{1}{r}$. Then for all functions $f, g \in \mathcal{S}(\mathbb{R})$, there exists a constant $C$ such that

$$
\|H(f, g)\|_{L^{p}(\mathbb{R})} \leq C\|f\|_{L^{p}(\mathbb{R})}\|g\|_{L^{q}(\mathbb{R})} .
$$

In this work the authors developed very powerful techniques known as timefrequency techniques and settled a longstanding conjecture of A.P. Calderón.

In this paper we are concerned with $L^{p}$ boundedness properties of bilinear Littlewood-Paley square functions on $\mathbb{R}^{d}$ and on $\mathbb{T}^{d}, d \geq 1$. The theory of linear Littlewood-Paley square functions is very interesting and has a wide range of applications in harmonic analysis. The study of Littlewood-Paley square functions

Received by the editors June 4, 2011.

2010 Mathematics Subject Classification. Primary 42A45, 42B15, 42B25.

Key words and phrases. Bilinear multipliers, Littlewood-Paley square functions. 
in the context of bilinear multipliers was initiated by Lacey [4] in 1996. As far as our knowledge is concerned, not much is known about bilinear square functions. We refer the interested reader to [1, 2, 3, 8, 9] for the work done so far (to our knowledge) on bilinear square functions.

Let $\omega$ be a cube in $\mathbb{R}^{d}, d \geq 1$. Then for $f, g \in S\left(\mathbb{R}^{d}\right)$ we can define a bilinear operator $S_{\omega}$ associated with the symbol $\chi_{\omega}(\xi-\eta)$ as follows:

$$
S_{\omega}(f, g)(x)=\int_{\mathbb{R}^{d}} \int_{\mathbb{R}^{d}} \hat{f}(\xi) \hat{g}(\eta) \chi_{\omega}(\xi-\eta) e^{2 \pi i x \cdot(\xi+\eta)} d \xi d \eta .
$$

When $d=1$, using Theorem 1.2 it is known that $S_{\omega}$ maps $L^{p}(\mathbb{R}) \times L^{q}(\mathbb{R})$ into $L^{r}(\mathbb{R})$, where $1<p, q \leq \infty$ and $\frac{2}{3}<r<\infty$ with $\frac{1}{p}+\frac{1}{q}=\frac{1}{r}$.

The bilinear square functions are defined as follows:

Definition 1.3 (Bilinear Littlewood-Paley Square Functions). Let $\left\{\omega_{l}\right\}_{l \in \mathbb{Z}^{d}}$ be a sequence of disjoint cubes in $\mathbb{R}^{d}$. Let $S_{\omega_{l}}$ be the bilinear operator associated with the symbol $\chi_{\omega_{l}}(\xi-\eta)$ as defined above. Then for $f, g \in S\left(\mathbb{R}^{d}\right)$, the bilinear LittlewoodPaley square function associated with the sequence $\left\{\omega_{l}\right\}_{l \in \mathbb{Z}^{d}}$ is defined as

$$
S(f, g)(x)=\left(\sum_{l \in \mathbb{Z}^{d}}\left|S_{\omega_{l}}(f, g)(x)\right|^{2}\right)^{\frac{1}{2}} .
$$

These square functions are referred to as non-smooth bilinear square functions.

The smooth bilinear square functions are defined similarly. More precisely, for a sequence of disjoint cubes $\omega_{l}$ in $\mathbb{R}^{d}$, a smooth bilinear square function is defined as

$$
T(f, g)(x):=\left(\sum_{l \in \mathbb{Z}^{d}}\left|T_{\phi_{l}}(f, g)(x)\right|^{2}\right)^{\frac{1}{2}}, f, g \in S\left(\mathbb{R}^{d}\right),
$$

where $T_{\phi_{l}}$ is the bilinear multiplier operator associated with the smooth symbol $\phi_{l}$ with $\operatorname{supp}\left(\phi_{l}\right) \subset \omega_{l}$.

Our interest is to obtain $L^{p}$ boundedness properties of bilinear square functions. As mentioned previously, the first result in this direction is due to Lacey. He proved the following:

Theorem $1.4(4)$. Let $\phi$ be a smooth function defined on $\mathbb{R}^{d}$ such that $\phi$ is supported in the unit cube of $\mathbb{R}^{d}$. For $l \in \mathbb{Z}^{n}$, let $\phi_{l}$ be the function defined by $\phi_{l}(\xi)=\phi(\xi-l)$. Then, for all $2 \leq p, q \leq \infty$ satisfying $\frac{1}{p}+\frac{1}{q}=\frac{1}{2}$, there exists a constant $C>0$ such that for all $f, g \in \mathcal{S}\left(\mathbb{R}^{d}\right)$ we have

$$
\|T(f, g)\|_{L^{2}\left(\mathbb{R}^{d}\right)} \leq C\|f\|_{L^{p}\left(\mathbb{R}^{d}\right)}\|g\|_{L^{q}\left(\mathbb{R}^{d}\right)} .
$$

The proof of the above theorem is quite intricate, and we notice that the exponent $r=2$ is crucial in the proof. In the same paper, Lacey posed the following natural questions about bilinear Littlewood-Paley square functions.

(1) Does Theorem 1.4 hold for $r \neq 2$ ?

(2) What about the non-smooth version of Theorem 1.4?

As one can easily see, the boundedness of non-smooth square functions on $\mathbb{R}$ implies the boundedness of the bilinear Hilbert transform on $\mathbb{R}$, which is known to be a very hard problem. So the second question about the non-smooth square function is supposed to be very difficult to answer. Bernicot [1] provided a positive 
result in this direction. In fact he proved much more in the form of the following theorem:

Theorem 1.5 ([1). Let $\left\{\omega_{n}=\left[a_{n}, b_{n}\right]\right\}_{n \in \mathbb{Z}}$ be a sequence of disjoint intervals in $\mathbb{R}$ with $b_{n}-a_{n}=b_{n-1}-a_{n-1}$ and $a_{n+1}-b_{n}=a_{n}-b_{n-1}$ for all $n \in \mathbb{Z}$. Then for exponents $2<p, q, r^{\prime}<\infty$ satisfying $\frac{1}{p}+\frac{1}{q}=\frac{1}{r}$, there is a constant $C=C(p, q)$ such that for all functions $f, g \in \mathcal{S}(\mathbb{R})$ we have

$$
\left\|\left(\sum_{n \in \mathbb{Z}}\left|S_{\omega_{n}}(f, g)\right|^{2}\right)^{\frac{1}{2}}\right\|_{L^{r}(\mathbb{R})} \leq C\|f\|_{L^{p}(\mathbb{R})}\|g\|_{L^{q}(\mathbb{R})} .
$$

The proof of the above theorem is very complicated. The author has developed suitable time-frequency techniques to deal with square functions. The condition that the intervals are of equal lengths and are equi-distant plays a very important role in the proof.

The first question of Lacey is answered in two papers: Mohanty and Shrivastava [8] and Bernicot and Shrivastava [2]. They proved that

Theorem $1.6([8,2])$. Let $\phi \in C^{\infty}(\mathbb{R})$ have support contained in the unit interval of $\mathbb{R}$. For $l \in \mathbb{Z}$, define $\phi_{l}(\xi)=\phi(\xi-l)$. Then for $2 \leq p, q \leq \infty$ and $1<r \leq \infty$ satisfying $\frac{1}{p}+\frac{1}{q}=\frac{1}{r}$, there exists a constant $C=C(\phi, p, q)>0$ such that for all $f, g \in \mathcal{S}(\mathbb{R})$, we have

$$
\left\|\left(\sum_{l \in \mathbb{Z}}\left|T_{\phi_{l}}(f, g)\right|^{2}\right)^{\frac{1}{2}}\right\|_{L^{r}(\mathbb{R})} \leq C\|f\|_{L^{p}(\mathbb{R})}\|g\|_{L^{q}(\mathbb{R})} .
$$

Mohanty and Shrivastava [8] proved the above theorem for exponents $\frac{4}{3}<r \leq \infty$. In order to prove the above theorem, the authors showed that the square function under consideration is dominated by the bilinear Hardy-Littlewood maximal function. Then using a celebrated result due to Lacey [5] about boundedness of the bilinear Hardy-Littlewood maximal operator, the authors deduced the required $L^{p}$ estimates for square functions. The use of the bilinear Hardy-Littlewood maximal function imposes the condition $r>\frac{4}{3}$. In the same paper, the authors also proved that for inequality (1.8) to hold true, condition $2 \leq p, q$ is necessary. Later, Bernicot and Shrivastava [2], using time-frequency techniques, proved $L^{p}$ estimates for a more general smooth bilinear square function. More precisely, they proved that

Theorem $1.7([2])$. Let $\Omega=\{\omega\}_{\omega_{\Omega}}$ be a well-distributed collection of intervals (i.e. $\sum_{\omega \in \Omega} \chi_{\omega} \leq C$ for some $C>0$ ) satisfying

$$
\inf _{\omega \in \Omega}|\omega| \simeq \sup _{\omega \in \Omega}|\omega| .
$$

Then, for $2 \leq p, q, r^{\prime}<\infty$ satisfying $\frac{1}{r}=\frac{1}{p}+\frac{1}{q}$, there exists a constant $C$, independent of the collection $\Omega$, such that for all $f, g \in \mathcal{S}(\mathbb{R})$, we have

$$
\left\|\left(\sum_{\omega \in \Omega}\left|T_{\psi_{\omega}}(f, g)\right|^{2}\right)^{1 / 2}\right\|_{L^{r}(\mathbb{R})} \leq C\|f\|_{L^{p}(\mathbb{R})}\|g\|_{L^{q}(\mathbb{R})},
$$

where $\psi_{\omega}$ is a smooth function supported in the interval $\omega$. 
As an application of the above theorem, the authors deduced Theorem 1.6 for the remaining exponents $1<r \leq \frac{4}{3}$. Thus, we see that for exponents $1<r \leq 2$ the proof of Theorem 1.6 relies on time-frequency techniques, which makes it very difficult. Also, for this range of exponents we do not have this theorem in higher dimensions. Moreover, the end point $r=1$ is not covered in Theorem 1.6.

The study of bilinear square functions on $\mathbb{T}$ was initiated by Mohanty and Shrivastava 9 . They proved the bilinear analogue of Carleson's Littlewood-Paley theorem on $\mathbb{T}$. Unlike the linear case, the bilinear Carleson's Littlewood-Paley theorem on $\mathbb{T}$ is not straightforward (for more details see [9]). The authors used suitable transference techniques in order to prove their result.

The purpose of this paper is to provide an elementary proof of Theorem 1.6 for the entire possible range of exponents, $2 \leq p, q \leq \infty, 1 \leq r \leq \infty$. We would like to remark that our proof works in the case of higher dimensions as well. Moreover, our proof is valid with a weaker hypothesis on the function $\phi$; see condition (2.1) with $\check{\phi}=K$, where we assume only a certain integrability condition on $K$. In this paper we also obtain $L^{p}$ estimates for bilinear Littlewood-Paley square functions on $\mathbb{T}^{d}$. As a consequence of this result, we obtain boundedness of the bilinear Carleson's Littlewood-Paley operator on $\mathbb{T}^{d}$.

\section{Smooth Bilinear Square Functions on $\mathbb{R}^{d}$}

In this section we study smooth bilinear square functions on $\mathbb{R}^{d}$ and give an easy proof of Theorem 1.6 in $\mathbb{R}^{d}$ for all possible values of the exponents $p, q, r$. In particular, we prove the following:

Theorem 2.1. Let $K$ be a measurable function on $\mathbb{R}^{d}$ such that

$$
\sum_{m \in \mathbb{Z}^{n}}\left(\int_{Q_{m}}|K(y)|^{\rho} d y\right)^{\frac{1}{\rho}}<\infty,
$$

where $\rho=\max \left\{2, r^{\prime}\right\}$ and $Q_{m}=\prod_{j=1}^{d}\left[m_{j}, m_{j}+1\right)$. For $l \in \mathbb{Z}^{n}$, define $\hat{K}_{l}(\xi)=$ $\hat{K}(\xi-l)$ and let $T_{l}$ be the bilinear operator associated with $\hat{K}_{l}(\xi-\eta)$. Then for exponents $2 \leq p, q \leq \infty$ and $1 \leq r \leq \infty$ satisfying $\frac{1}{p}+\frac{1}{q}=\frac{1}{r}$, there exists a constant $C=C(K, r)$ such that we have

$$
\left\|\left(\sum_{l \in \mathbb{Z}^{d}}\left|T_{l}(f, g)\right|^{2}\right)^{\frac{1}{2}}\right\|_{L^{r}\left(\mathbb{R}^{d}\right)} \leq C\|f\|_{L^{p}\left(\mathbb{R}^{d}\right)}\|g\|_{L^{q}\left(\mathbb{R}^{d}\right)} .
$$

Proof. For notational convenience we prove this theorem only in one dimension. The same proof is valid in higher dimensions as well.

We use the definition of $T_{l}(f, g)$ in terms of the kernel (see (1.1) ), i.e.,

$$
\begin{aligned}
T_{l}(f, g)(x) & =\int_{\mathbb{R}} f(x-y) g(x+y) K_{l}(y) d y \\
& =\int_{\mathbb{R}} f(x-y) g(x+y) e^{2 \pi i l y} K(y) d y .
\end{aligned}
$$


We claim that for almost every $x \in \mathbb{R}$ and for all $f, g \in \mathcal{S}(\mathbb{R})$, the square function satisfies the following pointwise estimate:

$$
\left(\sum_{l \in \mathbb{Z}}\left|T_{l}(f, g)(x)\right|^{2}\right)^{\frac{1}{2}} \leq \sum_{m \in \mathbb{Z}}\left(\int_{m}^{m+1}|f(x-y) g(x+y) K(y)|^{2} d y\right)^{\frac{1}{2}}
$$

Let $a=\left\{a_{l}\right\}_{l \in \mathbb{Z}}$ be a sequence in $l_{2}(\mathbb{Z})$ such that $\|a\|_{l_{2}(\mathbb{Z})}=1$. Then we prove that for a.e. $x \in \mathbb{R}$, we have

$$
\left|\sum_{l \in \mathbb{Z}} a_{l} T_{l}(f, g)(x)\right| \leq \sum_{m \in \mathbb{Z}}\left(\int_{m}^{m+1}|f(x-y) g(x+y) K(y)|^{2} d y\right)^{\frac{1}{2}} .
$$

Once we have the above estimate we can deduce the claimed estimate (2.3) using a duality argument. Consider

$$
\begin{aligned}
\sum_{l} a_{l} T_{l}(f, g)(x) & =\sum_{l} a_{l} \int_{\mathbb{R}} f(x-y) g(x+y) K_{l}(y) d y \\
& =\int_{\mathbb{R}} f(x-y) g(x+y) K(y) \sum_{l} a_{l} e^{2 \pi i l y} d y \\
& =\int_{\mathbb{R}} f(x-y) g(x+y) K(y) \hat{a}(y) d y
\end{aligned}
$$

where $\hat{a}$ is the Fourier transform of sequence $a$. Note that $\hat{a}$ is a periodic function such that $\|\hat{a}\|_{L^{2}([0,1])}=1$. Hence

$$
\begin{aligned}
\left|\sum_{l} a_{l} T_{l}(f, g)(x)\right| & \leq \sum_{m \in \mathbb{Z}} \int_{m}^{m+1}|f(x-y) g(x+y) K(y) \hat{a}(y)| d y \\
& \leq \sum_{m \in \mathbb{Z}}\left(\int_{m}^{m+1}|f(x-y) g(x+y) K(y)|^{2} d y\right)^{\frac{1}{2}}\left(\int_{m}^{m+1}|\hat{a}(y)|^{2} d y\right)^{\frac{1}{2}} \\
& =\sum_{m \in \mathbb{Z}}\left(\int_{m}^{m+1}|f(x-y) g(x+y) K(y)|^{2} d y\right)^{\frac{1}{2}} .
\end{aligned}
$$

This proves the claim.

We shall consider cases $1 \leq r \leq 2$ and $2 \leq r \leq \infty$ separately.

Case $1.1 \leq r \leq 2$.

From estimate (2.3) we have

$$
\begin{gathered}
\left\|\left(\sum_{l \in \mathbb{Z}}\left|T_{l}(f, g)\right|^{2}\right)^{\frac{1}{2}}\right\| \|_{L^{r}(\mathbb{R})} \leq \sum_{m \in \mathbb{Z}}\left[\int_{\mathbb{R}}\left(\int_{m}^{m+1}|f(x-y) g(x+y) K(y)|^{2} d y\right)^{\frac{r}{2}} d x\right]^{\frac{1}{r}} \\
=\sum_{m \in \mathbb{Z}}\left[\sum_{n \in \mathbb{Z}} \int_{n}^{n+1}\left(\int_{m}^{m+1}|f(x-y) g(x+y) K(y)|^{2} d y\right)^{\frac{r}{2}} d x\right]^{\frac{1}{r}} \\
\leq \sum_{m \in \mathbb{Z}}\left[\sum_{n \in \mathbb{Z}}\left(\int_{n}^{n+1} \int_{m}^{m+1}|f(x-y) g(x+y) K(y)|^{2} d y d x\right)^{\frac{r}{2}}\right]^{\frac{1}{r}} .
\end{gathered}
$$


Here we have used Hölder's inequality with exponents $\frac{2}{r}$ and $\left(\frac{2}{r}\right)^{\prime}$ as $1 \leq r \leq 2$. For $n, m \in \mathbb{Z}$, set $k_{m}(x)=\chi_{[m, m+1)}(x) K(x)$ and

$$
A_{n, m}=\int_{n}^{n+1} \int_{m}^{m+1}|f(x-y) g(x+y) K(y)|^{2} d y d x .
$$

Then,

$$
\begin{aligned}
A_{n, m} & =\int_{x=n}^{n+1} \int_{y=m}^{m+1}\left|f(x-y) g(x+y) k_{m}(y)\right|^{2} d y d x \\
& \leq \int_{z=n-m-1}^{n-m+1} \int_{w=n+m}^{n+m+2}\left|f(z) g(w) k_{m}\left(\frac{w-z}{2}\right)\right|^{2} \frac{d z d w}{2} \\
& =\int_{\mathbb{R}}\left|g_{n+m}(w)\right|^{2}\left(\int_{\mathbb{R}}\left|f_{n-m}(z) \tilde{k}_{m}(w-z)\right|^{2} d z\right) d w \\
& =\int_{\mathbb{R}}\left|g_{n+m}(w)\right|^{2}\left|f_{n-m}\right|^{2} *\left|\tilde{k}_{m}\right|^{2}(w) d w \\
& \leq\left\|g_{n+m}\right\|_{L^{q}(\mathbb{R})}^{2}\left\|f_{n-m}^{2} * \tilde{k}_{m}^{2}\right\|_{\left.L^{(q / 2)^{\prime}} \mathbb{R}\right)},
\end{aligned}
$$

where $\tilde{k}_{m}(x)=\frac{1}{\sqrt{2}} k_{m}\left(\frac{x}{2}\right), g_{n+m}(x)=\chi_{[n+m, n+m+2)}(x) g(x)$, and $f_{n-m}(x)=$ $\chi_{[n-m-1, n-m+1)}(x) f(x)$. We have used Hölder's inequality for exponents $\frac{q}{2}$ and $(q / 2)^{\prime}$ as $2 \leq q$.

Let $s=\frac{r^{\prime}}{2}$. Since $1 \leq r \leq 2$, we have $s \geq 1$. Using the condition $\frac{1}{p}+\frac{1}{q}=\frac{1}{r}$ we see that

$$
\frac{1}{p / 2}+\frac{1}{s}=1+\frac{1}{(q / 2)^{\prime}} .
$$

Hence using Young's inequality we get that

$$
\left\|f_{n-m}^{2} * \tilde{k}_{m}^{2}\right\|_{\left.L^{(q / 2)^{\prime}} \mathbb{R}\right)} \leq\left\|f_{n-m}^{2}\right\|_{L^{p / 2}(\mathbb{R})}\left\|\tilde{k}_{m}^{2}\right\|_{L^{s}(\mathbb{R})} .
$$

Substituting this estimate in the above we get

$$
\begin{aligned}
A_{n, m} & \leq\left\|g_{n+m}\right\|_{L^{q}(\mathbb{R})}^{2}\left\|f_{n-m}^{2}\right\|_{L^{p / 2}(\mathbb{R})}\left\|\tilde{k}_{m}^{2}\right\|_{L^{s}(\mathbb{R})} \\
& =\left\|\tilde{k}_{m}^{2}\right\|_{s}\left\|f_{n-m}\right\|_{p}^{2}\left\|g_{n+m}\right\|_{q}^{2} .
\end{aligned}
$$

Thus we have

$$
\begin{aligned}
\left\|\left(\sum_{l \in \mathbb{Z}}\left|T_{l}(f, g)\right|^{2}\right)^{\frac{1}{2}}\right\| \|_{L^{r}(\mathbb{R})} \leq \sum_{m \in \mathbb{Z}}\left[\sum_{n \in \mathbb{Z}}\left(\left\|\tilde{k}_{m}^{2}\right\|_{L^{s}(\mathbb{R})}\left\|f_{n-m}\right\|_{L^{p}(\mathbb{R})}^{2}\left\|g_{n+m}\right\|_{L^{q}(\mathbb{R})}^{2}\right)^{\frac{r}{2}}\right]^{\frac{1}{r}} \\
\leq \sum_{m \in \mathbb{Z}}\left\|\tilde{k}_{m}^{2}\right\|_{L^{s}(\mathbb{R})}^{\frac{1}{2}}\left(\sum_{n \in \mathbb{Z}}\left\|f_{n-m}\right\|_{L^{p}(\mathbb{R})}^{r}\left\|g_{n+m}\right\|_{L^{q}(\mathbb{R})}^{r}\right)^{\frac{1}{r}} \\
\leq \sum_{m \in \mathbb{Z}}\left\|k_{m}\right\|_{L^{r^{\prime}(\mathbb{R})}}\left(\sum_{n \in \mathbb{Z}}\left\|f_{n-m}\right\|_{L^{p}(\mathbb{R})}^{p}\right)^{\frac{r}{p}}\left(\sum_{n \in \mathbb{Z}}\left\|g_{n+m}\right\|_{L^{q}(\mathbb{R})}^{q}\right)^{\frac{r}{q}} \\
\leq C\left(\sum_{m \in \mathbb{Z}}\left\|k_{m}\right\|_{L^{r^{\prime}(\mathbb{R})}}\right)\|f\|_{L^{p}(\mathbb{R})}\|g\|_{L^{q}(\mathbb{R})} .
\end{aligned}
$$

This completes the proof of Theorem 2.1 for $1 \leq r \leq 2$. 
Case 2. $2 \leq r \leq \infty$.

In this case we use Minkowski's integral inequality for exponent $\frac{r}{2}$ as $2 \leq r$.

$$
\begin{aligned}
\left\|\left(\sum_{l \in \mathbb{Z}}\left|T_{l}(f, g)\right|^{2}\right)^{\frac{1}{2}}\right\|_{L^{r}(\mathbb{R})} & \leq \sum_{m \in \mathbb{Z}}\left[\int_{\mathbb{R}}\left(\int_{m}^{m+1}|f(x-y) g(x+y) K(y)|^{2} d y\right)^{\frac{r}{2}} d x\right]^{\frac{1}{r}} \\
& \leq \sum_{m \in \mathbb{Z}}\left[\int_{m}^{m+1}\left(\int_{\mathbb{R}}|f(x-y) g(x+y) K(y)|^{r} d x\right)^{\frac{2}{r}} d y\right]^{\frac{1}{2}} \\
& \leq \sum_{m \in \mathbb{Z}}\left[\int_{m}^{m+1}|K(y)|^{2}\left(\int_{\mathbb{R}}|f(x-y) g(x+y)|^{r} d x\right)^{\frac{2}{r}} d y\right]^{\frac{1}{2}} \\
& \leq \sum_{m \in \mathbb{Z}}\left(\int_{m}^{m+1}|K(y)|^{2} d y\right)^{\frac{1}{2}}\|f\|_{L^{p}(\mathbb{R})}\|g\|_{L^{q}(\mathbb{R})} .
\end{aligned}
$$

In order to get the second inequality we have used Hölder's inequality with exponents $\frac{p}{r}$ and $\frac{q}{r}$ as $\frac{1}{p}+\frac{1}{q}=\frac{1}{r}$. This completes the proof for the second case when $2 \leq r \leq \infty$.

\section{Bilinear square FunCtions on $\mathbb{T}^{d}$}

In this section we study bilinear square functions on $\mathbb{T}^{d}$. As mentioned previously, Mohanty and Shrivastava 9] proved that the bilinear Carleson's Littlewood-Paley operator maps $L^{p}(\mathbb{T}) \times L^{q}(\mathbb{T})$ into $L^{r}(\mathbb{T})$ for exponents $p, q, r$ satisfying $2 \leq p, q \leq \infty$ and the Hölder condition $\frac{1}{p}+\frac{1}{q}=\frac{1}{r}$. The authors used vector-valued transference methods to prove their result. In this section we observe that the arguments used in Section 2 to prove Theorem 2.1 can be applied to obtain boundedness of analogous bilinear square functions on $\mathbb{T}^{d}$. More precisely, we prove the following:

Theorem 3.1. Let $K$ be a measurable function on $\mathbb{T}^{d}$ such that $\|K\|_{L^{t}\left(\mathbb{T}^{d}\right)}<\infty$, where $t=\max \left\{2, r^{\prime}\right\}$. For $l \in \mathbb{Z}^{n}$, define $\hat{K}_{l}(n)=\hat{K}(n-l)$ and let $\tilde{S}_{l}$ be the bilinear multiplier operator associated with the symbol $\hat{K}_{l}(n-m)$. Then for exponents $2 \leq$ $p, q \leq \infty$ and $1 \leq r \leq \infty$ satisfying $\frac{1}{p}+\frac{1}{q}=\frac{1}{r}$, there exists a constant $C=C(K, \bar{r})$ such that for all trigonometric polynomials $f, g$ on $\mathbb{T}^{d}$, we have

$$
\left\|\left(\sum_{l \in \mathbb{Z}}\left|T_{l}(f, g)\right|^{2}\right)^{\frac{1}{2}}\right\|_{L^{r}\left(\mathbb{T}^{d}\right)} \leq C\|f\|_{L^{p}\left(\mathbb{T}^{d}\right)}\|g\|_{L^{q}\left(\mathbb{T}^{d}\right)} .
$$

Proof. The proof of this theorem follows using essentially the same arguments as in the previous section. We shall present here the main steps only. Also for convenience we work in dimension one only as the higher dimensional result follows similarly.

Let $f, g$ be trigonometric polynomials defined on $\mathbb{T}$. Then we have the following pointwise estimate for the square function (follow the proof of inequality (2.3)):

$$
\left(\sum_{l \in \mathbb{Z}}\left|\tilde{S}_{l}(f, g)(x)\right|^{2}\right)^{\frac{1}{2}} \leq\left(\int_{\mathbb{T}}|f(x-y) g(x+y) K(y)|^{2} d y\right)^{\frac{1}{2}} .
$$

Likewise as earlier we consider cases $1 \leq r<2$ and $2 \leq r \leq \infty$ separately. 
Case $1.1 \leq r \leq 2$.

Using estimate (3.2) and the condition that $1 \leq r<2$ we have

$$
\begin{aligned}
\left\|\left(\sum_{l \in \mathbb{Z}}\left|\tilde{S}_{l}(f, g)\right|^{2}\right)^{\frac{1}{2}}\right\|_{L^{r}(\mathbb{T})} & \leq\left[\int_{\mathbb{T}}\left(\int_{\mathbb{T}}|f(x-y) g(x+y) K(y)|^{2} d y\right)^{\frac{r}{2}} d x\right]^{\frac{1}{r}} \\
& \leq\left[\int_{\mathbb{T}} \int_{\mathbb{T}}|f(x-y) g(x+y) K(y)|^{2} d y d x\right]^{\frac{1}{2}} .
\end{aligned}
$$

We follow the proof of inequality (2.6) and obtain that

$$
\int_{\mathbb{T}} \int_{\mathbb{T}}|f(x-y) g(x+y) K(y)|^{2} d y d x \leq\left\|k_{2}^{2}\right\|_{L^{s}(\mathbb{T})}\|f\|_{L^{p}(\mathbb{T})}^{2}\|g\|_{L^{q}(\mathbb{T})}^{2},
$$

where $k_{2}(x)=\frac{1}{\sqrt{2}} K\left(\frac{x}{2}\right)$ and $s=\frac{r^{\prime}}{2}$. Putting the above two estimates together we have

$$
\left\|\left(\sum_{l \in \mathbb{Z}}\left|\tilde{S}_{l}(f, g)\right|^{2}\right)^{\frac{1}{2}}\right\|_{L^{r}(\mathbb{T})} \leq C\|K\|_{L^{r^{\prime}(\mathbb{T})}}\|f\|_{L^{p}(\mathbb{T})}\|g\|_{L^{q}(\mathbb{T})} .
$$

This completes the first case.

Case $2.2 \leq r \leq \infty$.

This part is easy as in the previous section. Since $2 \leq r \leq \infty$, using Minkowski's integral inequality we have

$$
\begin{aligned}
\left\|\left(\sum_{l \in \mathbb{Z}}\left|\tilde{S}_{l}(f, g)\right|^{2}\right)^{\frac{1}{2}}\right\|_{L^{r}(\mathbb{T})} & \leq\left[\int_{\mathbb{T}}\left(\int_{\mathbb{T}}|f(x-y) g(x+y) K(y)|^{2} d y\right)^{\frac{r}{2}} d x\right]^{\frac{1}{r}} \\
& \leq\left[\int_{\mathbb{T}}\left(\int_{\mathbb{T}}|f(x-y) g(x+y) K(y)|^{r} d x\right)^{\frac{2}{r}} d y\right]^{\frac{1}{2}} \\
& \leq\|K\|_{L^{2}(\mathbb{T})}\|f\|_{L^{p}(\mathbb{T})}\|g\|_{L^{q}(\mathbb{T})} .
\end{aligned}
$$

This completes the proof of Theorem 3.1

Remark 3.2. Let $\tilde{S}_{l}, l \in \mathbb{Z}^{d}$, denote the bilinear multiplier operator on $\mathbb{T}^{d}$ associated with the symbol $\chi_{\{l\}}(n-m)$. Consider the bilinear analogue of Carleson's Littlewood-Paley operator given by $(f, g) \rightarrow\left(\sum_{l \in \mathbb{Z}^{d}}\left|\tilde{S}_{l}(f, g)\right|^{2}\right)^{\frac{1}{2}}$. We know that $\chi_{\{l\}}(n)=\hat{K}(n-l)$, where $K \equiv 1$ on $[0,1]^{d}$. We can easily verify that $K$ satisfies the hypothesis of Theorem 3.1. Hence as an application of Theorem 3.1 we get that the bilinear Carleson's Littlewood-Paley operator maps $L^{p}\left(\mathbb{T}^{d}\right) \times L^{q}\left(\mathbb{T}^{d}\right)$ into $L^{r}\left(\mathbb{T}^{d}\right)$, where the exponents $p, q, r$ satisfy $2 \leq p, q \leq \infty, 1 \leq r \leq \infty$, and the Hölder condition $\frac{1}{p}+\frac{1}{q}=\frac{1}{r}$. 


\section{REFERENCES}

[1] Bernicot, F., $L^{p}$ estimates for non smooth bilinear Littlewood-Paley square functions on $\mathbb{R}$, Math. Ann. 351 (2011), no. 1, 1-49. MR2824844

[2] Bernicot, F., Shrivastava, S., Boundedness of smooth bilinear square functions and applications to some bilinear pseudo-differential operators. To appear in Indiana Univ. Math. J., available at arXiv:1010.1320v2 [math.CA].

[3] Diestel, G., Some remarks on bilinear Littlewood-Paley theory, J. Math. Anal. Appl. 307 (2005), 102-119. MR2138978 (2006j:42021)

[4] Lacey, M., On bilinear Littlewood-Paley square functions, Publ. Mat. 40 (1996), no. 2, 387-396. MR:1425626 (98c:42017)

[5] Lacey, M., The bilinear maximal functions map into $L^{p}$ for $2 / 3<p \leq 1$, Ann. of Math. (2) 151 (2000), no. 1, 35-57. MR1745019 (2001b:42015)

[6] Lacey, M., Thiele, C., $L^{p}$ estimates on the bilinear Hilbert transform for $2<p<\infty$, Ann. of Math. (2) 146 (1997), no. 3, 693-724. MR.1491450 (99b:42014)

[7] Lacey, M., Thiele, C., On Calderón's conjecture, Ann. of Math. (2) 149 (1999), no. 2, 475-496. MR.1689336 (2000d:42003)

[8] Mohanty, P., Shrivastava, S., A note on the bilinear Littlewood-Paley square function, Proc. Amer. Math. Soc. 138 (2010), no. 6, 2095-2098. MR2596047 (2011b:42065)

[9] Mohanty, P., Shrivastava, S., Bilinear Littlewood-Paley for circle and transference, Publ. Math. 55 (2011), no. 2, 501-519. MR2839453

School of Mathematics, Harish-Chandra Research Institute, Allahabad, India E-mail address: ratnapk@hri.res.in

School of Mathematics, Harish-Chandra Research Institute, Allahabad, India E-mail address: saurabhkumar@hri.res.in 